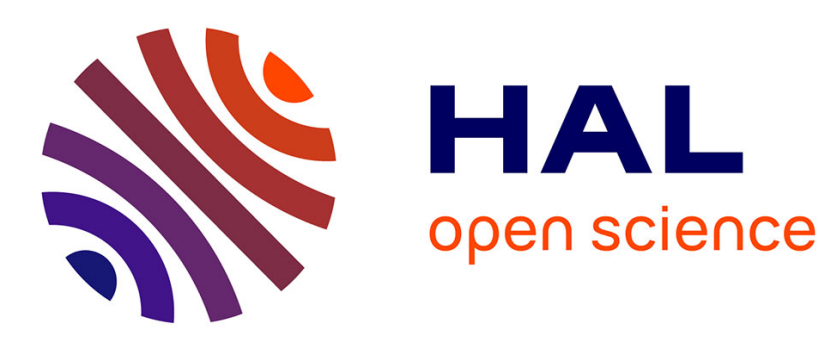

\title{
Amortized bivariate multi-point evaluation
}

Joris van Der Hoeven, Grégoire Lecerf

\section{To cite this version:}

Joris van Der Hoeven, Grégoire Lecerf. Amortized bivariate multi-point evaluation. International Symposium on Symbolic and Algebraic Computation 2021, Jul 2021, Saint Petersbourg, Russia. 10.1145/3452143.3465531. hal-03124458

\section{HAL Id: hal-03124458 \\ https://hal.science/hal-03124458}

Submitted on 28 Jan 2021

HAL is a multi-disciplinary open access archive for the deposit and dissemination of scientific research documents, whether they are published or not. The documents may come from teaching and research institutions in France or abroad, or from public or private research centers.
L'archive ouverte pluridisciplinaire HAL, est destinée au dépôt et à la diffusion de documents scientifiques de niveau recherche, publiés ou non, émanant des établissements d'enseignement et de recherche français ou étrangers, des laboratoires publics ou privés. 


\title{
Amortized bivariate multi-point evaluation ${ }^{* \dagger}$
}

\author{
JORIS VAN DER HOEVEN ${ }^{a b}$, GRÉGOIRE LECERF ${ }^{a c}$ \\ a. CNRS, École polytechnique, Institut Polytechnique de Paris \\ Laboratoire d'informatique de l'École polytechnique (LIX, UMR 7161) \\ 1 , rue Honoré d'Estienne d'Orves \\ Bâtiment Alan Turing, CS35003 \\ 91120 Palaiseau, France \\ b. Email: vdhoeven@lix.polytechnique.fr \\ c. Email: lecerf@lix.polytechnique.fr \\ Preliminary version of January 28, 2021
}

\begin{abstract}
The evaluation of a polynomial at several points is called the problem of multi-point evaluation. Sometimes, the set of evaluation points is fixed and several polynomials need to be evaluated at this set of points. Efficient algorithms for this kind of "amortized" multi-point evaluation were recently developed for the special case when the set of evaluation points is sufficiently generic. In this paper, we design a new algorithm for arbitrary sets of points, while restricting ourselves to bivariate polynomials.
\end{abstract}

\section{INTRODUCTION}

Let $\mathbb{K}$ be an effective field, so that we have algorithms for the field operations. Given a polynomial $P \in \mathbb{K}\left[x_{1}, \ldots, x_{D}\right]$ and a tuple $\alpha=\left(\alpha_{1}, \ldots, \alpha_{n}\right) \in\left(\mathbb{K}^{D}\right)^{n}$ of points, the computation of $P(\boldsymbol{\alpha})=\left(P\left(\alpha_{1}\right), \ldots, P\left(\alpha_{n}\right)\right) \in \mathbb{K}^{n}$ is called the problem of multi-point evaluation. The converse problem is called interpolation and takes a candidate support of $P$ as input.

These problems naturally occur in several areas of applied algebra. When solving a polynomial system, multi-point evaluation can for instance be used to check whether all points in a given set are indeed solutions of the system. In [12], we have shown that fast algorithms for multi-point evaluation actually lead to efficient algorithms for polynomial system solving. The more specific problem of bivariate multi-point evaluation appears for example in the computation of generator matrices of algebraic geometry error correcting codes [15].

The general problem of multivariate multi-point evaluation is notoriously hard. If $\mathbb{K}=\mathbb{Q}$ or $\mathbb{K}$ is a field of finite characteristic, then theoretical algorithms with quasioptimal bit complexity exponent are due to Kedlaya and Umans [14]. Unfortunately, to our best knowledge, these algorithms do not seem suitable for practical purposes [11, Conclusion]. We also mention [17] for a general algorithm in the bivariate case and [13] for an efficient algorithm in the case of special sets of points $\alpha$.

Last year, new softly linear algorithms have been proposed for multi-point evaluation and interpolation in the case when $\alpha$ is a fixed generic tuple of points $[10,16]$. These algorithms are amortized in the sense that potentially expensive precomputations as a function of $\boldsymbol{\alpha}$ are allowed. The algorithms from [10] work in arbitrary dimension, whereas [16] is restricted to the bivariate case.

*. This paper is part of a project that has received funding from the French "Agence de l'innovation de défense".

†. This article has been written using GNU TE $\mathrm{X}_{\text {MACS }}[8]$. 
In the present paper, we turn our attention to arbitrary (i.e. possibly non-generic) tuples of evaluation points $\alpha$, while restricting ourselves to the bivariate case $D=2$. Combining ideas from [10] and [16], we present a new softly linear algorithm for amortized multi-point evaluation. We have not optimized all constant factors involved in the cost analysis of our new algorithm, so our complexity bound is mostly of theoretical interest for the moment. The opposite task of interpolation is more subtle: since interpolants of total degree $O(\sqrt{n})$ do not necessarily exist, the very problem needs to be stated with additional care. For this reason, we do not investigate multi-point interpolation in this paper.

\section{WEIGHTED BIVARIATE POLYNOMIALS}

Our bivariate multi-point evaluation makes use of polynomial arithmetic with respect to weighted graded monomial orderings. This section is devoted to the costs of products and divisions in this context.

\subsection{Complexity model}

For complexity analyses, we will only consider algebraic complexity models like computation trees [2], for which elements in $\mathbb{K}$ are freely at our disposal. The time complexity simply measures the number of arithmetic operations and zero-tests in $\mathbb{K}$.

We denote by $\mathrm{M}(d)$ the time that is needed to compute a product $P Q$ of two polynomials $P, Q \in \mathbb{K}[x]$ of degree $<d$. We make the usual assumptions that $\mathrm{M}(d) / d$ is non-decreasing as a function of $d$ and that $\mathrm{M}(k d)=O(k \mathrm{M}(d))$ whenever $k=O(d)$. Using a variant of the Schönhage-Strassen algorithm [3], it is well known that $M(d)=$ $O(d \log d \log \log d)$. If we restrict our attention to fields $\mathbb{K}$ of positive characteristic, then we may even take $\mathrm{M}(d)=O\left(d \log d 4^{\log ^{*} d}\right)$ [5].

\subsection{Monomial orderings}

General monomial orderings, that are suitable for Gröbner basis computations, have been classified in [18]. For the purpose of this paper, we focus on the following specific family of bivariate monomial orderings.

DEFINITION 1. Let $k \in \mathbb{N} \backslash\{0\}$. We define the $k$-degree of a monomial $x^{a} y^{b}$ with $a, b \in \mathbb{N}$ by

$$
\operatorname{deg}_{k}\left(x^{a} y^{b}\right):=a+k b \text {. }
$$

We define the $k$-ordering to be the monomial ordering $\prec_{k}$ such that

$$
x^{a} y^{b} \prec_{k} x^{u} y^{v} \Leftrightarrow\left\{\begin{array}{l}
a+k b<u+k v \\
a+k b=u+k v \text { and } b<v .
\end{array}\right. \text { or }
$$

Let us mention that the idea of using such kinds of families of monomial orderings in the design of fast algorithms for bivariate polynomials previously appeared in [9].

\subsection{Multiplication}

Consider the product $C=A B$ of two non-zero bivariate polynomials $A, B \in \mathbb{K}[x, y]$ and the obvious bound

$$
s:=\left(\operatorname{deg}_{x} A+\operatorname{deg}_{x} B+1\right)\left(\operatorname{deg}_{y} A+\operatorname{deg}_{y} B+1\right)
$$

for the number of terms of $C$. Then it is well known that Kronecker substitution allows for the computation of the product $C$ using $O(M(s))$ operations in $\mathbb{K}$; see [4, Corollary 8.27], for instance. 
Writing val $x A$ for the valuation of $A$ in $x$, the number of non-zero terms of $C$ is more accurately bounded by

$$
\tilde{s}:=\left(\operatorname{deg}_{x} A+\operatorname{deg}_{x} B-\operatorname{val}_{x} A-\operatorname{val}_{x} B+1\right)\left(\operatorname{deg}_{y} A+\operatorname{deg}_{y} B+1\right) .
$$

Via the appropriate multiplications and divisions by powers of $x$, we observe that $C$ can be computed using $O(\mathrm{M}(\tilde{s}))$ operations in $\mathbb{K}$.

Let us next show that a similar bound holds for slices of polynomials that are dense with respect to the $k$-ordering. More precisely, let $\operatorname{val}_{k} A$ denote the minimum of $i+k j$ over the monomials $x^{i} y^{j}$ occurring in $A$. By convention we set $\operatorname{val}_{k} 0:=+\infty$. From the monomial identity

$$
x^{i}\left(x^{k} y\right)^{j}=x^{i+k j} y^{j}
$$

we observe that the monomials of $k$-degree $d$ are in one-to-one correspondence with the monomials of degree $d$ in $x$ and degree in $y$ in $\{0, \ldots,\lfloor d / k\rfloor\}$. It also follows that the number of terms of a non-zero polynomial $A$ is bounded by

$$
\left(\operatorname{deg}_{k} A-\operatorname{val}_{k} A+1\right)\left(\operatorname{deg}_{y} A+1\right),
$$

and that

$$
\begin{aligned}
\operatorname{val}_{x}\left(A\left(x, x^{k} y\right)\right) & =\operatorname{val}_{k} A \\
\operatorname{deg}_{x}\left(A\left(x, x^{k} y\right)\right) & =\operatorname{deg}_{k} A .
\end{aligned}
$$

Then, the number of non-zero terms in the product $C=A B$ is bounded by

$$
s_{k}:=\left(\operatorname{deg}_{k} A+\operatorname{deg}_{k} B-\operatorname{val}_{k} A-\operatorname{val}_{k} B+1\right)\left(\operatorname{deg}_{y} A+\operatorname{deg}_{y} B+1\right) .
$$

LEMMA 2. The above product $C=A B$ can be computed with $O\left(\mathrm{M}\left(s_{k}\right)\right)$ operations in $\mathbb{K}$.

Proof. It suffices to compute $C$ using

$$
C\left(x, x^{k} y\right)=A\left(x, x^{k} y\right) B\left(x, x^{k} y\right)
$$

and to apply the cost bound from above to this computation.

\subsection{Division}

Let $B$ be a polynomial in $\mathbb{K}[x, y]$ of $k$-degree $\delta$ and of leading monomial written $x^{\alpha} y^{\beta}$. Without loss of generality we may assume that the coefficient of this monomial is 1 . We examine the cost of the division of $A$ of $k$-degree $d$ by $B$ with respect to $\prec_{k}$ :

$$
A=Q B+R,
$$

where $Q$ and $R$ are in $\mathbb{K}[x, y]$, and such that no monomial in $R$ is divisible by $x^{\alpha} y^{\beta}$. Such a division does exist: this is a usual fact from the theory of Gröbner bases. In this context, a polynomial $A$ is said to be reduced with respect to $B$ when none of its terms is divisible by $x^{\alpha} y^{\beta}$.

If $A=\tilde{Q} B+\tilde{R}$ for polynomials $\tilde{Q}$ and $\tilde{R}$ such that $\tilde{R}$ is reduced with respect to $B$, then $(Q-\tilde{Q}) B=\tilde{R}-R$, so $\tilde{Q}=Q$ and $\tilde{R}=R$. In other words, $Q$ and $R$ are unique, so we may write quo $_{k}(A, B)$ for the quotient $Q$ of $A$ by $B$ with respect to $\prec_{k}$.

In the remainder of this section, we assume that $k$ has been fixed once and for all. Given $A=\sum_{(i, j) \in \mathbb{N}^{2}} A_{i, j} x^{i} y^{j} \in \mathbb{K}[x, y]$, we define

$$
A_{[a]}:=\sum_{i+k j=a} A_{i, j} x^{i} y^{j}, \quad A_{(a, b]}:=\sum_{a<i+k j \leqslant b} A_{i, j} x^{i} y^{j} .
$$


The naive division algorithm proceeds as follows: if $A$ has a term $A_{i, j} x^{i} y^{j}$ that is divisible by $x^{\alpha} y^{\beta}$, then we pick a maximal such term for $\prec_{k}$ and compute

$$
\tilde{A}:=A-A_{i, j} x^{i-\alpha} y^{j-\beta} B \text {. }
$$

Then $\operatorname{deg}_{k} \tilde{A} \leqslant \operatorname{deg}_{k} A$ and the largest term of $\tilde{A}$ that is divisible by $x^{\alpha} y^{\beta}$ is strictly less than $x^{i} y^{j}$ for $\prec_{k}$. This division step is repeated for $\tilde{A}$ and for its successive reductions, until $Q$ and $R$ are found.

During this naive division process, we note that $Q_{(d-\delta-l, d-\delta]}$ only depends on $A_{(d-l, d]}$ and $B_{(\delta-l, \delta]}$, for $l=0, \ldots, d-\delta+1$. When $l=0$ nothing needs to be computed. Let us now describe a more efficient way to handle the case $l=1$, when we need to compute the quasi-homogeneous component of $Q$ of maximal $k$-degree $d-\delta$ :

$$
A_{[d]}=Q_{[d-\delta]} B_{[\delta]}+R_{[d]} .
$$

LEMMA 3. We may compute $Q_{[d-\delta]}$ and $R_{[d]}$ using $O\left(\mathrm{M}\left(\operatorname{deg}_{y} A\right)\right)$ operations in $\mathbb{K}$.

Proof. We first decompose

$$
A_{[d]}=H+T, \quad H:=\sum_{\substack{i+k j=d \\ i \geqslant \alpha}} A_{i, j} x^{i} y^{j}, \quad T:=\sum_{\substack{i+k j=d \\ i<\alpha}} A_{i, j} x^{i} y^{j}
$$

and note that $T$ is reduced with respect to $B$. In particular, the division of $A_{[d]}$ by $B_{[\delta]}$ yields the same quotient as the division of $H$ by $B_{[\delta]}$, so

$$
\begin{aligned}
H & =Q_{[d-\delta]} B_{[\delta]}+U \\
R_{[d]} & =T+U,
\end{aligned}
$$

for some quasi-homogeneous polynomial $U$ of $k$-degree $d$ with $\operatorname{deg}_{x} U<\alpha$. Dehomogenization of the relation (1) yields

$$
H(1, y)=Q_{[d-\delta]}(1, y) B_{[\delta]}(1, y)+U(1, y),
$$

with $\operatorname{deg} U(1, y)<\beta$. Consequently, the computation of $Q_{[d-\delta]}(1, y)$ and $U(1, y)$ takes $O\left(\mathrm{M}\left(\operatorname{deg}_{y} A\right)\right)$ operations in $\mathbb{K}$, using a fast algorithm for Euclidean division in $\mathbb{K}[y]$; see [4, chapter 9] or [6], for instance.

For higher values of $l$, the following "divide and conquer" division algorithm is more efficient than the naive algorithm:

\section{Algorithm 1}

Input. $A, B \in \mathbb{K}[x, y]$ and an integer $l \in\{0, \ldots, d-\delta+1\}$, where $d:=\operatorname{deg}_{k} A$ and $\delta:=\operatorname{deg}_{k} B$.

Output. quo $_{k}(A, B)_{(d-\delta-l, d-\delta]}$.

1. If $d<\delta$ or $l=0$ then return 0 .

2. If $l=1$ then compute and return $\mathrm{quo}_{k}(A, B)_{[d-\delta]}$ using the method from Lemma 3.

3. Let $h:=\lfloor l / 2\rfloor$.

4. Recursively compute $Q_{1}:=$ quo $_{k}(A, B)_{(d-\delta-h, d-\delta]}$.

5. Let $R_{1}:=\left(A_{(d-l, d]}-Q_{1} B_{(\delta-l, \delta]}\right)_{(d-l, d-h]}$.

6. Recursively compute $Q_{0}:=\mathrm{quo}_{k}\left(R_{1}, B\right)_{(d-\delta-l, d-\delta-h]}$.

7. Return $Q_{1}+Q_{0}$. 
PROPOSITION 4. Algorithm 1 is correct and takes $O(\mathrm{M}(l d / k) \log l)$ operations in $\mathbb{K}$.

Proof. Let us prove the correctness by induction on $l$. If $d<\delta$, then quo $_{k}(A, B)=0$ and the result of the algorithm is correct. If $l=0$, then $\mathrm{quo}_{k}(A, B)_{(d-\delta-l, d-\delta]}=0$ and the result is also correct. The case $l=1$ has been treated in Lemma 3 .

Now assume that $l \geqslant 2$ and $d \geqslant \delta$, so $l>h \geqslant 1$. The induction hypothesis implies that $\left(A-Q_{1} B\right)_{(d-h, d]}$ is reduced with respect to $B$ and that $\left(R_{1}-Q_{0} B\right)_{(d-l, d-h]}$ is reduced with respect to $B$. After noting that

$$
\begin{aligned}
R_{1} & =\left(A_{(d-l, d]}-Q_{1} B_{(\delta-l, \delta]}\right)_{(d-l, d-h]} \\
& =\left(A-Q_{1} B\right)_{(d-l, d-h]},
\end{aligned}
$$

we verify that

$$
\begin{aligned}
\left(A-\left(Q_{1}+Q_{0}\right) B\right)_{(d-l, d]} & =\left(A-Q_{1} B\right)_{(d-l, d-h]}+\left(A-Q_{1} B\right)_{(d-h, d]}-\left(Q_{0} B\right)_{(d-l, d]} \\
& =R_{1}-\left(Q_{0} B\right)_{(d-l, d]}+\left(A-Q_{1} B\right)_{(d-h, d]} \\
& =R_{1}-\left(Q_{0} B\right)_{(d-l, d-h]}+\left(A-Q_{1} B\right)_{(d-h, d]} \\
& =\left(R_{1}-Q_{0} B\right)_{(d-l, d-h]}+\left(A-Q_{1} B\right)_{(d-h, d]} .
\end{aligned}
$$

Consequently, $\left(A-\left(Q_{1}+Q_{0}\right) B\right)_{(d-l, d]}$ is reduced with respect to $B$, whence

$$
Q_{1}+Q_{0}=\mathrm{quo}_{k}(A, B)_{(d-\delta-l, d-\delta]} .
$$

This completes the induction and our correctness proof.

Concerning the complexity, step 2 takes $O\left(\mathrm{M}\left(\operatorname{deg}_{y} A\right)\right)=O(\mathrm{M}(d / k))$ operations in $\mathbb{K}$, by Lemma 3. In step 5, the computation of $R_{1}$ takes $O\left(\mathrm{M}\left(l \operatorname{deg}_{y} A\right)\right)=O(\mathrm{M}(l d / k))$ operations in $\mathbb{K}$, by Lemma 2.

Let $T(\hat{d}, \hat{l})$ stand for the maximum of the costs of Algorithm 1 for $d \leqslant \hat{d}$ and $l \leqslant \hat{l}$. We have shown that $\mathrm{T}(d, 1)=O(\mathrm{M}(d / k))$ and that

$$
\begin{aligned}
\mathrm{T}(d, l) & \leqslant \mathrm{T}(d, h)+\mathrm{T}(d-h, l-h)+O(\mathrm{M}(l d / k)) \\
& \leqslant \mathrm{T}(d, h)+\mathrm{T}(d, l-h)+O(\mathrm{M}(l d / k)) .
\end{aligned}
$$

Unrolling this inequality, we obtain the claimed complexity bound.

Remark 5. The complexity bound from Proposition 4 is also a consequence of [7, Theorem 4] by taking $\mathrm{SM}(s):=O(\mathrm{M}(s))$ for the cost of sparse polynomial products of size $s$. This cost is warranted mutatis mutandis by the observation that all sparse bivariate polynomial products occurring within the algorithm underlying [7, Theorem 4] are either univariate products or products of slices of polynomials that are dense with respect to the $k$-ordering. We have seen in section 2.3 how to compute such products efficiently.

\section{GENERAL POSITION}

Let $\alpha=\left(\alpha_{1}, \ldots, \alpha_{n}\right) \in\left(\mathbb{K}^{2}\right)^{n}$ be a tuple of pairwise distinct points. We define the vanishing ideal for $\alpha$ by

$$
I_{\boldsymbol{\alpha}}:=\{P \in \mathbb{K}[x, y]: P(\boldsymbol{\alpha})=(0, \ldots, 0)\} .
$$

A monic polynomial $P \in I_{\alpha}$ is said to be axial if its leading monomial with respect to $\prec_{1}$ is of the form $y^{d}$. The goal of this section is to prove the existence of such a polynomial modulo a sufficiently generic change of variables of the form

$$
x=\tilde{x}+\lambda y .
$$


This change of variables transforms $\boldsymbol{\alpha}$ into a new tuple $\tilde{\boldsymbol{\alpha}} \in\left(\mathbb{K}^{2}\right)^{n}$ with

$$
\alpha_{i}=(x, y) \Longrightarrow \tilde{\alpha}_{i}=(x-\lambda y, y)
$$

and the ideal $I_{\alpha}$ into

$$
I_{\tilde{\boldsymbol{\alpha}}}:=\{\tilde{P} \in \mathbb{K}[\tilde{x}, y]: \tilde{P}(\tilde{\boldsymbol{\alpha}})=(0, \ldots, 0)\} .
$$

For any degree $d \in \mathbb{N}$, we define

$$
\mathbb{K}[x, y]_{\leqslant d}:=\left\{P \in \mathbb{K}[x, y]: \operatorname{deg}_{1} P \leqslant d\right\} .
$$

Given a polynomial $P \in \mathbb{K}[x, y]_{\leqslant d}$ such that $\operatorname{deg}_{1} P=d$ we may decompose

$$
P=D+R,
$$

where $D \in \mathbb{K}[x, y]$ is homogeneous of degree $d$ and $R \in \mathbb{K}[x, y]_{\leqslant d-1}$. The change of variables (3) transforms $P$ into

$$
\tilde{P}(\tilde{x}, y):=\tilde{D}(\tilde{x}, y)+\tilde{R}(\tilde{x}, y),
$$

where

$$
\begin{aligned}
\tilde{R}(\tilde{x}, y) & :=R(\tilde{x}+\lambda y, y) \in \mathbb{K}[\tilde{x}, y]_{\leqslant d-1}, \\
\tilde{D}(\tilde{x}, y) & :=D(\tilde{x}+\lambda y, y) .
\end{aligned}
$$

The coefficient of the monomial $y^{d}$ in $\tilde{D}(\tilde{x}, y)$ is $\tilde{D}(0,1)=D(\lambda, 1)$.

The $\mathbb{K}$-vector space $I_{\alpha} \cap \mathbb{K}[x, y]_{\leqslant d}$ is the solution set of a linear system consisting of $n$ equations and $\left(\begin{array}{c}d+2 \\ 2\end{array}\right)$ unknowns, that are the unknown coefficients of a polynomial in $\mathbb{K}[x, y]_{\leqslant d}$. Such a system admits a non-zero solution whenever $\left(\begin{array}{c}d+2 \\ 2\end{array}\right)>n$. Now assume that $P$ is a non-zero element of $I_{\alpha}$ of minimal total degree $d$ and let $\Lambda_{\alpha}$ denote the set of roots of $D(\lambda, 1)$ in $\mathbb{K}$. Since $d$ is minimal we have

that implies

$$
\left(\begin{array}{c}
d+1 \\
2
\end{array}\right)=\frac{d(d+1)}{2} \leqslant n
$$

$$
d \leqslant \sqrt{2 n}
$$

On the other hand, we have $\left|\Lambda_{\alpha}\right| \leqslant d$. And if $\lambda \in \mathbb{K} \backslash \Lambda_{\alpha}$, then $y^{d}$ is the leading monomial of $\tilde{P}$ for $\prec_{1}$. Assuming that $|\mathbb{K}|>n$, this proves the existence of an axial polynomial $\tilde{P}$ of degree $d$ after a suitable change of variables of the form (3).

We say that $\alpha$ is in general position if there exists a polynomial of minimal degree $d$ in $I_{\alpha}$ that is axial.

\section{POlyNOMIAL REDUCTION}

Let $\alpha=\left(\alpha_{1}, \ldots, \alpha_{n}\right) \in\left(\mathbb{K}^{2}\right)^{n}$ be a tuple of points in general position, as defined in the previous section. Given a polynomial $P \in \mathbb{K}[x, y]$, this section is devoted to a reduction process that computes a polynomial in $P+I_{\alpha}$ whose support is "relatively smaller" than the one of $P$.

\subsection{Heterogeneous bases}

Since $\boldsymbol{\alpha}$ is in general position, thanks to (4), we first precompute an axial polynomial $B_{0} \in I_{\alpha}$ for $\prec_{1}$ of degree

$$
\delta_{0}:=\operatorname{deg}_{1} B_{0} \leqslant \sqrt{2 n}
$$


For $i \geqslant 1$, let $N\left(d, 2^{i}\right)$ denote the number of monomials of $2^{i}$-degree $\leqslant d$. We have

$$
N\left(d, 2^{i}\right)=d+1+\left(d+1-2^{i}\right)+\cdots+\left(d+1-\left\lfloor\frac{d}{2^{i}}\right\rfloor 2^{i}\right) .
$$

If $N\left(d, 2^{i}\right)>n$, then there exists a non-zero polynomial in $I_{\alpha}$ of $2^{i}$-degree $\leqslant d$. Let $B_{i} \in I_{\alpha}$ be a monic polynomial whose leading monomial is minimal for $\prec_{2^{i}}$ and set

$$
\delta_{i}:=\operatorname{deg}_{2^{i}} B_{i} .
$$

We may precompute $B_{i}$, e.g. by extracting it from a Gröbner basis for $I_{\alpha}$ with respect to $\prec_{2}$. By the minimality of the $2^{i}$-degree $\delta_{i}$ of $B_{i}$, we have

$$
N\left(\delta_{i}-1,2^{i}\right) \leqslant n \text {. }
$$

Now write $\delta_{i}=q 2^{i}+r$ with $q \in \mathbb{N}$ and $r \in\left\{0, \ldots, 2^{i}-1\right\}$. Then

$$
\begin{aligned}
N\left(\delta_{i}-1,2^{i}\right) & =\left(q 2^{i}+r\right)+\left((q-1) 2^{i}+r\right)+\cdots+\left(2^{i}+r\right)+r \\
& =(q+\cdots+1) 2^{i}+(q+1) r \\
& =\frac{1}{2}(q+1) q 2^{i}+(q+1) r \\
& =\frac{1}{2}(q+1)\left(q 2^{i}+2 r\right) \\
& =\frac{1}{2^{i+1}}\left(q 2^{i}+2^{i}\right)\left(q 2^{i}+2 r\right) \\
& =\frac{1}{2^{i+1}}\left(\delta_{i}+2^{i}-r\right)\left(\delta_{i}+r\right) \\
& \geqslant \frac{1}{2^{i+1}} \delta_{i}^{2} .
\end{aligned}
$$

Consequently, $\delta_{i}^{2} \leqslant 2^{i+1} n$ and

$$
\delta_{i} \leqslant \sqrt{2^{i+1} n} .
$$

We let $\ell$ be the smallest integer such that $2^{\ell}>n$, hence

$$
2^{\ell-1} \leqslant n
$$

and $\ell:=\left\lceil\log _{2}(n+1)\right\rceil$. There exists a monic non-zero polynomial $Q$ in $\mathbb{K}[x] \cap I_{\alpha}$ of minimal degree $\leqslant n$. Since $2^{\ell}>n$, we may take $B_{i}:=Q$ for $i \geqslant \ell$. We call $\left(B_{i}\right)_{i \geqslant 0}$ a heterogeneous basis for $\boldsymbol{\alpha}$. We further define

$$
h_{i}:=\operatorname{deg}_{y} B_{i} \leqslant\left\lfloor\sqrt{2^{1-i} n}\right\rfloor .
$$

Note that $\ell$ is the first integer such that $h_{\ell+1}=0$, although $\operatorname{deg}_{y} B_{i}=0$ holds even for $i=\ell$.

\subsection{Elementary reductions}

Given $A=\sum_{\alpha, \beta} A_{\alpha, \beta} x^{\alpha} y^{\beta} \in \mathbb{K}[x, y]$ and $i \geqslant 0$, we may use the division procedure from section 2.4 to reduce $A$ with respect to $B_{i}$. This yields a relation

$$
A=Q B_{i}+R,
$$

where $\operatorname{deg}_{2^{i}} R \leqslant \operatorname{deg}_{2^{i}} A$ and such that none of the monomials in the support of $R$ is divisible by the leading monomial of $B_{i}$. We write $\rho_{i}(A):=R$ and recall that $\rho_{i}$ is a $\mathbb{K}$-linear map.

We also define the projections $\pi_{i}$ and $\bar{\pi}_{i}$ by

$$
\begin{aligned}
& \pi_{i}(A):=\sum_{\alpha \in \mathbb{N}, \beta \leqslant h_{i}} A_{\alpha, \beta} x^{\alpha} y^{\beta} \\
& \bar{\pi}_{i}(A):=\sum_{\alpha \in \mathbb{N}, \beta>h_{i}} A_{\alpha, \beta} x^{\alpha} y^{\beta} .
\end{aligned}
$$




\subsection{Compound reductions}

For $d \geqslant 1$, we let $\mathbb{K}[x, y]_{d}^{*}$ denote the set of tuples of polynomials $\left(P_{0}, \ldots, P_{m}\right) \in \mathbb{K}[x, y]^{m+1}$ such that

- $m$ is the first integer such that $2^{m}>d$,

- $\operatorname{deg}_{2^{i}} P_{i} \leqslant \sqrt{2^{i} d}$, for $i=0, \ldots, m$.

Intuitively speaking, such a tuple will represent a sum $P=P_{0}+\cdots+P_{m}$ modulo $I_{\alpha}$. Note that $\operatorname{deg}_{y} P_{m} \leqslant\left\lfloor\sqrt{2^{-m} d}\right\rfloor=0$, so $P_{m} \in \mathbb{K}[x]$.

Given $\left(P_{0}, \ldots, P_{m}\right) \in \mathbb{K}[x, y]^{m+1}$, we define three new sequences of polynomials by

$$
\begin{aligned}
& R_{0}:=\rho_{0}\left(P_{0}\right) \\
& \Pi_{0}:=\pi_{0}\left(R_{0}\right) \\
& \bar{\Pi}_{0}:=\bar{\pi}_{0}\left(R_{0}\right) \\
& R_{1}:=\rho_{1}\left(P_{1}+\Pi_{0}\right) \\
& \Pi_{1}:=\pi_{1}\left(R_{1}\right) \\
& \bar{\Pi}_{1}:=\bar{\pi}_{1}\left(R_{1}\right) \\
& R_{2}:=\rho_{2}\left(P_{2}+\Pi_{1}\right) \\
& \Pi_{2}:=\pi_{2}\left(R_{2}\right) \\
& \bar{\Pi}_{2}:=\bar{\pi}_{2}\left(R_{2}\right) \\
& : \\
& R_{m-1}:=\rho_{m-1}\left(P_{m-1}+\Pi_{m-2}\right) \\
& R_{m}:=\rho_{m}\left(P_{m}+\Pi_{m-1}\right) \text {. } \\
& \Pi_{m-1}:=\pi_{m-1}\left(R_{m-1}\right) \\
& \bar{\Pi}_{m-1}:=\bar{\pi}_{m-1}\left(R_{m-1}\right)
\end{aligned}
$$

LEMMA 6. With the above notations. Let $\beta \geqslant 16$ and let $\eta:=\left(1+\sqrt{\frac{2}{\beta}}\right)^{2}$. If $d \geqslant \beta n$, then

$$
\begin{aligned}
\operatorname{deg}_{2^{i}} R_{i} & \leqslant \sqrt{2^{i} d} \\
\operatorname{deg}_{2^{i+1}} \Pi_{i} & \leqslant \sqrt{2^{i} \eta d}
\end{aligned}
$$

for $i=0, \ldots, m-1$.

Proof. We first note that $\eta \leqslant 2$ and

$$
\sqrt{2^{i} d}+\sqrt{2^{i+1} n} \leqslant \sqrt{2^{i} \eta d}
$$

Let us prove the degree bounds by induction on $i=0, \ldots, m-1$. For $i=0$, we have

$$
\begin{aligned}
& \operatorname{deg}_{1} R_{0} \leqslant \operatorname{deg}_{1} P_{0} \leqslant \sqrt{d} \\
& \operatorname{deg}_{2} \Pi_{0} \leqslant \operatorname{deg}_{1} R_{0}+\operatorname{deg}_{y} \Pi_{0} \leqslant \operatorname{deg}_{1} R_{0}+h_{0} \leqslant \sqrt{d}+\sqrt{2 n} \leqslant \sqrt{\eta d},
\end{aligned}
$$

by using (7) and (9). Assume now that $0<i \leqslant m-1$ and that the bounds of the lemma hold for all smaller $i$. Since $\eta \leqslant 2$, the induction hypothesis yields

$$
\operatorname{deg}_{2^{i}} R_{i} \leqslant \max \left(\operatorname{deg}_{2^{i}} P_{i}, \operatorname{deg}_{2^{i}} \Pi_{i-1}\right) \leqslant \sqrt{2^{i} d} .
$$

Using (7) and (9) again, we deduce

$$
\operatorname{deg}_{2^{i+1}} \Pi_{i} \leqslant \operatorname{deg}_{2^{i}} R_{i}+2^{i} \operatorname{deg}_{y} \Pi_{i} \leqslant \operatorname{deg}_{2^{i}} R_{i}+h_{i} 2^{i} \leqslant \sqrt{2^{i} d}+\sqrt{2^{i+1} n} \leqslant \sqrt{2^{i} \eta d} .
$$

LEMMA 7. Under the assumptions of Lemma 6, we further have

$$
\bar{\Pi}_{1}+\cdots+\bar{\Pi}_{m-1}+R_{m}=P_{0}+\cdots+P_{m}+I_{\alpha} .
$$

Assume that $\theta:=\frac{1}{\sqrt{2}}+\frac{2}{\sqrt{\beta}}<1$, let $\tilde{d}:=\theta^{2} d$, and let $\tilde{m}$ be the first integer such that $2^{\tilde{m}}>\tilde{d}$. If $\tilde{m}=m$, then

$$
\left(\bar{\Pi}_{1}, \ldots, \bar{\Pi}_{m-1}, R_{m}, 0\right) \in \mathbb{K}[x, y]_{\tilde{d}}^{*} ;
$$

otherwise, $\tilde{m}=m-1$ and

$$
\left(\bar{\Pi}_{1}, \ldots, \bar{\Pi}_{m-1}, R_{m}\right) \in \mathbb{K}[x, y]_{\tilde{d}}^{*}
$$


Proof. By construction, we have

$$
R_{0}+\cdots+R_{m} \in P_{0}+\cdots+P_{m}+\Pi_{0}+\cdots+\Pi_{m-1}+I_{\alpha \prime}
$$

whence

$$
\begin{aligned}
\bar{\Pi}_{0}+\cdots+\bar{\Pi}_{m-1}+R_{m} & =\left(R_{0}-\Pi_{0}\right)+\cdots+\left(R_{m-1}-\Pi_{m-1}\right)+R_{m} \\
& \in P_{0}+\cdots+P_{m}+I_{\alpha} .
\end{aligned}
$$

Since $B_{0}$ is axial, we have $\bar{\Pi}_{0}=0$, which entails (10). From Lemma 6 we deduce

$$
\operatorname{deg}_{y} \bar{\Pi}_{i} \leqslant \frac{\operatorname{deg}_{2^{i}} \bar{\Pi}_{i}}{2^{i}} \leqslant \frac{\operatorname{deg}_{2^{i}} R_{i}}{2^{i}} \leqslant \frac{\sqrt{2^{i} d}}{2^{i}}=\sqrt{2^{-i} d} .
$$

Now $R_{i}$ contains no monomial that is divisible by the leading monomial of $B_{i}$ for $\prec_{2^{i}}$ and $\operatorname{deg}_{y} \bar{\Pi}_{i}>\operatorname{deg}_{y} B_{i}$. Using (5), it follows that

$$
\operatorname{deg}_{x} \bar{\Pi}_{i} \leqslant \operatorname{deg}_{x} B_{i} \leqslant \delta_{i} \leqslant \sqrt{2^{i+1} n} .
$$

Consequently, for $i=1, \ldots, m-1$, inequalities (11) and (12), combined with $d \geqslant \beta n$, lead to

$$
\begin{aligned}
\operatorname{deg}_{2^{i-1}} \bar{\Pi}_{i} & \leqslant \operatorname{deg}_{x} \bar{\Pi}_{i}+2^{i-1} \operatorname{deg}_{y} \bar{\Pi}_{i} \\
& \leqslant \sqrt{2^{i+1} n}+\sqrt{2^{i-2} d} \\
& \leqslant \theta \sqrt{2^{i-1} d}=\sqrt{2^{i-1} \tilde{d}} .
\end{aligned}
$$

From $d \geqslant \beta n \geqslant 16 n$ and (6), we deduce that $d \geqslant 2^{4} \times 2^{\ell-1}=2^{\ell+3}$, whence $m \geqslant \ell+4$. If follows that

$$
\operatorname{deg}_{y} \Pi_{m-1} \leqslant h_{m-1} \leqslant h_{\ell+3}=0 .
$$

From $\operatorname{deg}_{y} P_{m}=0$, we thus obtain $\operatorname{deg}_{y} R_{m}=0$ and $\operatorname{deg}_{x} R_{m}<n$.

Since $n \leqslant d / 16$ and $d<2^{m}$, we further deduce

$$
\begin{aligned}
\operatorname{deg}_{2^{m-1}} R_{m} & =\operatorname{deg}_{x} R_{m} \\
& <\sqrt{2^{-8} d^{2}} \\
& \leqslant \sqrt{2^{-7} \times 2^{m-1} d} \\
& \leqslant \theta \sqrt{2^{m-1} d}=\sqrt{2^{m-1} \tilde{d}} .
\end{aligned}
$$

Finally $d>\tilde{d} \geqslant d / 2$ and $2^{m-1} \leqslant d<2^{m}$ imply $2^{m-2} \leqslant \tilde{d}<d$, whence $\tilde{m} \in\{m-1, m\}$.

We call the tuple $\left(\bar{\Pi}_{1}, \ldots, \bar{\Pi}_{m-1}, R_{m}\right)$ the reduction of $\left(P_{0}, \ldots, P_{m}\right)$ with respect to $\left(B_{i}\right)_{i \geqslant 0}$.

LEMMA 8. With the above notation, the reduction of $\left(P_{0}, \ldots, P_{m}\right)$ with respect to $\left(B_{i}\right)_{i \geqslant 0}$ takes $O\left(\mathrm{M}(d) \log ^{2} d\right)$ operations in $\mathbb{K}$.

Proof. Note first that $\left(\operatorname{deg}_{1} P_{0}\right)^{2} \leqslant d$. Using Lemma 6, we also have

$$
\left(\operatorname{deg}_{2^{i}}\left(P_{i}+\Pi_{i-1}\right)\right)^{2} / 2^{i} \leqslant\left(\sqrt{2^{i} d}\right)^{2} / 2^{i}=d,
$$

for $i=1, \ldots, m$. By Proposition 4, each evaluation of $\rho_{i}$ takes $O(\mathrm{M}(d) \log d)$ operations in $\mathbb{K}$. The claimed bound follows from $m=O(\log d)$.

PROPOSITION 9. Let $\left(P_{i}\right)_{i \geqslant 0} \in \mathbb{K}[x, y]_{128 n}^{*}$. Then a sequence $\left(Q_{i}\right)_{i \geqslant 0} \in \mathbb{K}[x, y]_{64 n}^{*}$ with $\sum_{i \geqslant 0} Q_{i}=$ $\sum_{i \geqslant 0} P_{i}+I_{\alpha}$ can be computed using $O\left(\mathrm{M}(n) \log ^{2} n\right)$ operations in $\mathbb{K}$. 
Proof. We set $\beta:=64$, so that $\theta=\frac{1}{\sqrt{2}}+\frac{2}{\sqrt{\beta}}<0.958$. We set $\left(P_{i}^{[0]}\right)_{i \geqslant 0}:=\left(P_{i}\right)_{i \geqslant 0}$ and define $\left(P_{i}^{[k+1]}\right)_{i \geqslant 0}$ recursively as the reduction of $\left(P_{i}^{[k]}\right)_{i \geqslant 0}$ with respect to $\left(B_{i}\right)_{i \geqslant 0}$, for $k \geqslant 0$. We further define $d^{[k]}:=\theta^{2 k} 128 n$. The integer $K:=8$ is the first integer such that

$$
\theta^{2 K} \leqslant \frac{1}{2}
$$

We finally take $\left(Q_{i}\right)_{i \geqslant 0}:=\left(P_{i}^{[K]}\right)_{i \geqslant 0}$. Lemma 7 implies that $\left(Q_{i}\right)_{i \geqslant 0}$ belongs to $\mathbb{K}[x, y]_{64 n}^{*}$. The complexity bound follows from Lemma 8.

\section{Multi-POint EVAlUAtion}

Let $\alpha \in\left(\mathbb{K}^{2}\right)^{n}$ be a tuple of pairwise distinct points and consider the problem of fast multipoint evaluation of a polynomial $P \in \mathbb{K}[x, y]$ of total degree $<\sqrt{2 n}$ at $\alpha$. For simplicity of the exposition, it is convenient to first consider the case when $n=2^{v}$ is a power of two and $\alpha$ is in general position. The core of our method is based on the usual "divide and conquer" paradigm.

We say that $\alpha$ is in recursive general position if $\alpha$ is in general position and if $\boldsymbol{\alpha}_{1, n / 2}:=$ $\left(\alpha_{1}, \ldots, \alpha_{n / 2}\right)$ and $\boldsymbol{\alpha}_{n / 2+1, n}:=\left(\alpha_{n / 2+1}, \ldots, \alpha_{n}\right)$ are in recursive general position. An empty sequence is in recursive general position. With the notation of section 3 a recursive general position is ensured if the cardinality of $\mathbb{K}$ is strictly larger that $\lambda_{n}$ that is recursively defined by

$$
\lambda_{n}:=n+2 \lambda_{n / 2}
$$

for $n \geqslant 4$ and with $\lambda_{2}:=2$. Consequently $\lambda_{n}=n v$. Whenever $|\mathbb{K}|>n v$, we know from section 3 that we can reduce to the case where $\alpha$ is in recursive general position. In this case, we can compute a recursive heterogeneous basis, that is made of a heterogeneous basis for $\boldsymbol{\alpha}$ and recursive heterogeneous bases for $\boldsymbol{\alpha}_{1, n / 2}$ and $\boldsymbol{\alpha}_{n / 2+1, n}$.

\section{Algorithm 2}

Input. $\alpha \in\left(\mathbb{K}^{2}\right)^{n}$ with $n=2^{v}$ and $\left(P_{i}\right)_{i \geqslant 0} \in \mathbb{K}[x, y]_{128 n}^{*}$.

Output. $\left(\sum_{i \geqslant 0} P_{i}\right)(\boldsymbol{\alpha})$.

Precomputed. A recursive heterogeneous basis for $\alpha$.

Assumption. $\alpha$ is in recursive general position.

1. If $v=0$, then return $\left(\sum_{i \geqslant 0} P_{i}\right)\left(\alpha_{1}\right)$.

2. Compute the reduction $\left(Q_{i}\right)_{i \geqslant 0} \in \mathbb{K}[x, y]_{64 n}^{*}$ of $\left(P_{i}\right)_{i \geqslant 0}$ with respect to the heterogeneous basis $\left(B_{i}\right)_{i \geqslant 0}$ for $\boldsymbol{\alpha}$, via Proposition 9 .

3. Recursively apply the algorithm to $\alpha_{1, n / 2}$ and $\left(Q_{i}\right)_{i \geqslant 0}$.

4. Recursively apply the algorithm to $\boldsymbol{\alpha}_{n / 2+1, n}$ and $\left(Q_{i}\right)_{i \geqslant 0}$.

5 . Return the concatenations of the results of the recursive evaluations.

THEOREM 10. Algorithm 2 is correct and runs in time $O\left(M(n) \log ^{3} n\right)$.

Proof. The algorithm is clearly correct if $v=0$. If $v>0$, then we first observe that both $\boldsymbol{\alpha}_{1, n / 2}$ and $\boldsymbol{\alpha}_{n / 2+1, n}$ are in recursive general position by definition. Furthermore, Proposition 9 ensures that

$$
\left(\sum_{i \geqslant 0} P_{i}\right)(\boldsymbol{\alpha})=\left(\sum_{i \geqslant 0} Q_{i}\right)(\boldsymbol{\alpha}) .
$$


The concatenation of the results of the recursive applications of the algorithm therefore yields the correct result.

As to the complexity bound, the cost of step 2 is bounded by $O\left(\operatorname{M}(n) \log ^{2} n\right)$ according to Proposition 9. Hence, the total execution time $\mathrm{T}(n)$ satisfies

$$
\mathrm{T}(n) \leqslant 2 \mathrm{~T}\left(\frac{n}{2}\right)+O\left(\mathrm{M}(n) \log ^{2} n\right) .
$$

The desired complexity bound follows by unrolling this recurrence inequality.

COROLLARY 11. Consider an arbitrary effective field $\mathbb{K}$ and $\boldsymbol{\alpha} \in\left(\mathbb{K}^{2}\right)^{n}$, where $n$ is not necessarily a power of two. Modulo precomputations that only depend on $\mathbb{K}$ and $\boldsymbol{\alpha}$, we can evaluate any polynomial in $\mathbb{K}[x, y]_{\sqrt{2 n}}$ at $\boldsymbol{\alpha}$ in time $O\left(\mathrm{M}(n) \log ^{4} n\right)$.

Proof. Modulo the repetition of at most $n-1$ more points, we may assume without loss of generality that $n$ is a power of two $2^{v}$.

If $\mathbb{K}$ is finite then we build an algebraic extension $\mathbb{E}$ of $\mathbb{K}$ of degree $e:=O(\log n)$, so that $|\mathbb{E}|>n v$. Multiplying two polynomials in $\mathbb{E}[x]_{\leqslant n}$ takes $O(\mathrm{M}(e n))=O(e \mathrm{M}(n))=$ $O(\mathrm{M}(n) \log n)$ operations in $\mathbb{K}$ thanks to our assumptions on $\mathrm{M}$. Consequently, up to introducing an extra $\log n$ factor in our complexity bounds, we may assume that $|\mathbb{K}|>n v$. Modulo a change of variables (3) from section 3, we may then assume without loss of generality that $\alpha$ is in recursive general position, and compute a recursive heterogeneous basis.

Given $P \in \mathbb{K}[x, y]_{\sqrt{2 n}}$, we claim that we may compute $\tilde{P}(\tilde{x}, y):=P(\tilde{x}+\lambda y, y)$ using $O(\mathrm{M}(n) \log n)$ operations in $\mathbb{K}$. Indeed, we first decompose

$$
P(x, y)=p_{0}(x, y)+\cdots+p_{m}(x, y),
$$

where $m \leqslant \sqrt{2 n}$ and each $p_{i}$ is zero or homogenous of degree $i$. Computing $p_{i}(\tilde{x}+\lambda y, y)$ then reduces to computing $p_{i}(\tilde{x}+\lambda, 1)$. This corresponds in turn to a univariate Taylor shift, which takes $O(\mathrm{M}(i) \log i)$ operations in $\mathbb{K}$; see [1, Lemma 7], for instance.

Finally, we apply Theorem 10 to $(\tilde{P}, 0, \ldots) \in \mathbb{K}[\tilde{x}, y]_{128 n}^{*}$ and $\tilde{\boldsymbol{\alpha}}$.

\section{BIBLIOGRAPHY}

[1] J. Berthomieu, G. Lecerf, and G. Quintin. Polynomial root finding over local rings and application to error correcting codes. Appl. Alg. Eng. Comm. Comp., 24(6):413-443, 2013.

[2] P. Bürgisser, M. Clausen, and M. A. Shokrollahi. Algebraic Complexity Theory, volume 315 of Grundlehren der Mathematischen Wissenschaften. Springer-Verlag, 1997.

[3] D. G. Cantor and E. Kaltofen. On fast multiplication of polynomials over arbitrary algebras. Acta Inform., 28:693-701, 1991.

[4] J. von zur Gathen and J. Gerhard. Modern Computer Algebra. Cambridge University Press, 2nd edition, 2002.

[5] D. Harvey and J. van der Hoeven. Faster polynomial multiplication over finite fields using cyclotomic coefficient rings. J. Complexity, 54:101404, 2019.

[6] J. van der Hoeven. Newton's method and FFT trading. J. Symbolic Comput., 45(8):857-878, 2010.

[7] J. van der Hoeven. On the complexity of multivariate polynomial division. In I. S. Kotsireas and E. Martínez-Moro, editors, Applications of Computer Algebra. Kalamata, Greece, July 20-23, 2015, volume 198 of Springer Proceedings in Mathematics E Statistics, pages 447-458. Cham, 2017. Springer International Publishing.

[8] J. van der Hoeven. The Jolly Writer. Your Guide to GNU TeXmacs. Scypress, 2020.

[9] J. van der Hoeven and R. Larrieu. Fast reduction of bivariate polynomials with respect to sufficiently regular Gröbner bases. In C. Arreche, editor, Proceedings of the 2018 ACM International Symposium on Symbolic and Algebraic Computation, ISSAC '18, pages 199-206. New York, NY, USA, 2018. ACM. 
[10] J. van der Hoeven and G. Lecerf. Fast amortized multi-point evaluation. Technical Report, HAL, 2020. https://hal . archives-ouvertes.fr/hal-02508529.

[11] J. van der Hoeven and G. Lecerf. Fast multivariate multi-point evaluation revisited. J. Complexity, 56:101405, 2020.

[12] J. van der Hoeven and G. Lecerf. On the complexity exponent of polynomial system solving. Found. Comput. Math., 2020. https://doi .org/10.1007/s10208-020-09453-0.

[13] J. van der Hoeven and É. Schost. Multi-point evaluation in higher dimensions. Appl. Alg. Eng. Comm. Comp., 24(1):37-52, 2013.

[14] K. S. Kedlaya and C. Umans. Fast polynomial factorization and modular composition. SIAM J. Comput., 40(6):1767-1802, 2011.

[15] D. Le Brigand and J.-J. Risler. Algorithme de Brill-Noether et codes de Goppa. Bulletin de la société mathématique de France, 116(2):231-253, 1988.

[16] V. Neiger, J. Rosenkilde, and G. Solomatov. Generic bivariate multi-point evaluation, interpolation and modular composition with precomputation. In A. Mantzaflaris, editor, Proceedings of the 45 th International Symposium on Symbolic and Algebraic Computation, ISSAC '20, pages 388-395. New York, NY, USA, 2020. ACM.

[17] M. Nüsken and M. Ziegler. Fast multipoint evaluation of bivariate polynomials. In S. Albers and T. Radzik, editors, Algorithms - ESA 2004. 12th Annual European Symposium, Bergen, Norway, September 14-17, 2004, volume 3221 of Lect. Notes Comput. Sci., pages 544-555. Springer Berlin Heidelberg, 2004.

[18] L. Robbiano. Term orderings on the polynomial ring. In B. F. Caviness, editor, EUROCAL '85. European Conference on Computer Algebra. Linz, Austria, April 1-3, 1985. Proceedings. Volume 2: Research Contributions, volume 204 of Lect. Notes Comput. Sci., pages 513-517. Springer-Verlag Berlin Heidelberg, 1985. 